\title{
Angular correlations in the prompt neutron emission in spontaneous fission of ${ }^{252} \mathrm{Cf}$
}

\author{
Yuri Kopatch ${ }^{1, \text { a }}$, Andreina Chietera ${ }^{2}$, Louise Stuttgé ${ }^{2}$, Friedrich Gönnenwein ${ }^{3}$, Manfred Mutterer $^{4,5}$, Alexei Gagarski ${ }^{6}$, \\ Irina Guseva $^{6}$, Olivier Dorvaux ${ }^{2}$, Francis Hanappe ${ }^{7}$, and Franz-Josef Hambsch ${ }^{8}$ \\ 1 Joint Institute for Nuclear Research (JINR), Joliot Curie 6, 141980 Dubna, Moscow region, Russia \\ 2 IPHC, Université de Strasbourg, Strasbourg, France CNRS, UMR7178, 67037 Strasbourg, France \\ 3 Physikalisches Institut, Universität Tübingen, 72076 Tübingen, Germany \\ ${ }^{4}$ Institut für Kernphysik, Technische Universität, 64289 Darmstadt, Germany \\ 5 GSI Helmholtzzentrum für Schwerionenforschung, 64291 Darmstadt, Germany \\ ${ }^{6}$ Petersburg Nuclear Physics Institute, 188300 Gatchina, Russia \\ 7 PNTPM, Université Libre de Bruxelles, 1050 Bruxelles, Belgium \\ 8 European Commission (EC), Joint Research Centre (JRC), Institute for Reference Materials and Measurements (IRMM), 2440 Geel, \\ Belgium
}

\begin{abstract}
An experiment aiming at the detailed investigation of angular correlations in the neutron emission from spontaneous fission of ${ }^{252} \mathrm{Cf}$ has been performed at IPHC Strasbourg using the angle-sensitive double ionization chamber CODIS for measuring fission fragments and a set of 60 DEMON scintillator counters for neutron detection. The main aim of the experiment is to search for an anisotropy of neutron emission in the center-of-mass system of the fragments. The present status of the data analysis and the full Monte-Carlo simulation of the experiment are reported in the present paper.
\end{abstract}

\section{Introduction}

It is known since decades that in the laboratory reference frame the neutron emission from fission fragments (FF) is anisotropic relative to the fission axis with more neutrons being emitted along this axis [1,2]. The experimentally observed anisotropy is rather well explained by kinematical focusing of neutrons evaporated isotropically in the center-of mass system $(\mathrm{cms})$ of fully equilibrated and accelerated fragments. However, at closer examination a small but significant discrepancy between experiment and theory is revealed which cannot be explained by uncertainties in the calculation of neutron evaporation or focusing [3]. Two possible reasons are proposed to solve the discrepancy: a) the existence of so-called "scission neutrons" [4] emitted at the moment of scission with an angular distribution differing in the laboratory frame from the one by evaporation from fully accelerated fragments and b) the anisotropy of neutron emission in the cms of fragments due to a correlation between neutron emission and angular momentum of fragments (dynamical anisotropy) [5,6]. It is well established that for $\gamma$-ray emission the fragment spins being aligned perpendicular to the fission axis induce anisotropy relative to the fission axis [7]. For neutron evaporation the angular distribution in the cms depends on the angular momentum $l$ carried away in the emission. Neutrons emitted with non-zero angular momenta $l>0$ are preferentially ejected perpendicular to FF spin in the cms. Since FF spin itself is perpendicular to the fission axis,

a e-mail: kopatch@nf .jinr.ru anisotropy appears also relative to the fission axis in the $\mathrm{cms}$ with emission being favoured along the fission axis. Hence the kinematical and the dynamical anisotropy in the Lab system have the same sign and it is not evident how to disentangle the small dynamical from the dominant kinematical contribution. The new idea proposed by $\mathrm{F}$. Gönnenwein is to exploit the fact that, in contrast to kinematics, in the dynamical anisotropy the neutrons from a cascade are correlated in angle due to their preferred emission in the same plane perpendicular to fragment spin. It is therefore proposed to measure coincidences between two successive neutrons and a fission fragment from the same event. For analysis these events are projected on a plane perpendicular to the fission axis. For dynamical anisotropy the difference in azimuthal angles between two neutrons are in this plane preferentially $0^{\circ}$ or $180^{\circ}$, while kinematical anisotropy produces a huge isotropic background. Note that scission neutrons will not simulate correlated events.

\section{Experiment}

The experiment was performed at the IPHC laboratory in Strasbourg. The experimental setup "CORA" consists of a $4 \pi$ detector for fission fragments CODIS [8], surrounded by a set of 60 DEMON liquid scintillator cells [9] for measuring prompt fission neutrons (see Fig. 1).

The spectrometer CODIS is a Frisch-gridded $4 \pi$ twin ionization chamber sharing a common cathode. The spontaneous fission ${ }^{252} \mathrm{Cf}$ source is mounted in the center of the cathode. A particular feature of the chamber is the cathode being divided into sectors. This allows measuring 


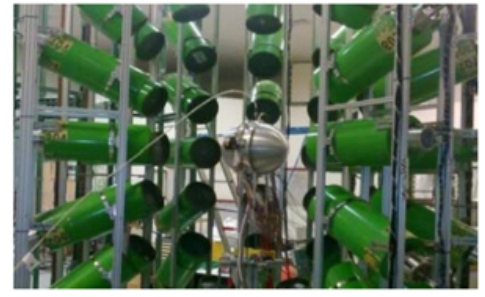

Figure 1. The experimental setup: DEMON detectors surrounding the CODIS chamber.
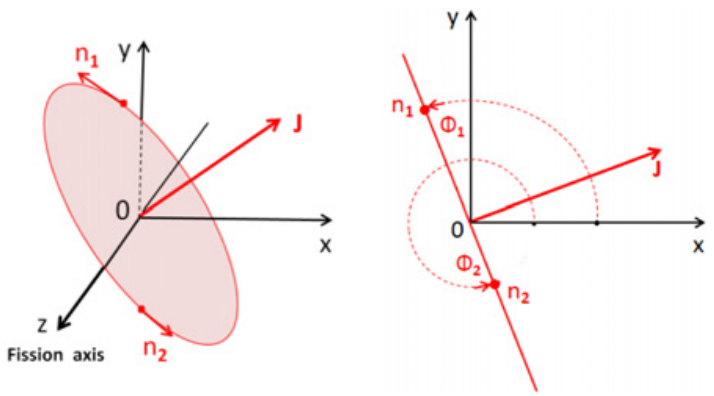

Figure 2. Schematic view of the neutron-spin angular correlations. Left: fission axis is along the $Z$-axis, fragment spins are aligned in a plane $X Y$, perpendicular to the fission axis; neutrons are emitted in a plane perpendicular to the fragment spin. Right: projection of the neutron emission directions onto the $X Y$ plane, perpendicular to the fission axis.

both, the azimuthal $\Phi$-angles and the polar angles $\theta$ of ion tracks in the chamber. Alternatively, the polar angles $\theta$ relative to the chamber axis are found by measuring the electron drift times to the Frisch grids.

We applied the following method for the analysis of the influence of fragment angular momentum (spin) on neutron emission from the fragments. All events are analyzed in a coordinate system, in which the $Z$ axis coincides with the flight direction of the light fission fragment, while the most probable orientation of the fragment spin $J$ is in the $X Y$ plane (see Fig. 2).

Two neutrons emitted from the same fragment with azimuthal angles $\Phi_{1}$ and $\Phi_{2}$ in the $X Y$ plane are considered. Since each neutron is correlated with fragment spin $J$, i.e. emitted preferentially perpendicular to spin, the two neutrons will also be correlated in angle $\Phi$. So one should observe a non-isotropic distribution of relative angles $\Phi_{21}=\left(\Phi_{2}--\Phi_{1}\right)$. Figure 2 visualizes the extreme case with fragment spin strictly perpendicular to the fission axis and neutron emission strictly in a plane perpendicular to spin. In this case, in the projection of the neutron emission pattern onto the $X Y$ plane all neutrons from the same fission event will be located on one single line. Thus, the difference in azimuthal angle $\Phi_{21}=\left(\Phi_{2}--\Phi_{1}\right)$ will be either $0^{\circ}$ or $180^{\circ}$ (see top in Fig. 3). Closer to reality the distribution $W\left(\Phi_{n n}\right)$ of relative neutron-neutron azimuthal angles $\Phi_{n n}$ in the $X Y$ plane perpendicular to the fission axis should have the following dependence [10]:

$$
W\left(\Phi_{n n}\right)=p_{0}\left(1+a_{2} \cos ^{2} \Phi_{n n}\right) .
$$

Unfortunately the estimated anisotropy factor $\mathrm{a}_{2}=\left[W\left(0^{\circ}\right)\right.$ $\left.W\left(90^{\circ}\right)\right] / W\left(90^{\circ}\right)$ in the $X Y$ plane is very small: $a_{2} \approx 0.003$ [10]. As shown by red dots in Fig 3 (bottom) the estimated anisotropy is a challenge for experiment.
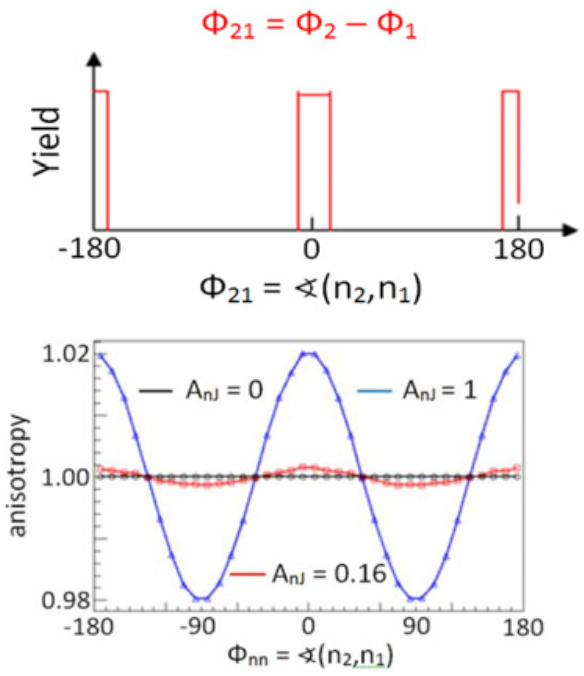

Figure 3. Distribution of the relative neutron-neutron angle $\Phi_{n n}$ in the plane perpendicular to the fission axis. Top panel: distribution $W\left(\Phi_{n n}\right)$ for extreme anisotropy; bottom panel: anisotropy of $\Phi_{n n}$ for different anisotropies $A_{n J}$ of neutron emission relative to fission axis (see chapter 3 ).

\section{Monte Carlo simulations}

A Monte-Carlo code was written to investigate the influence of detector properties of DEMON on experimental results. The code reproduces the DEMON detector configuration for analyzing the impact of the modular detector assembly on the geometrical acceptance and hence neutron angular distribution. It also addresses the interaction processes of neutrons in the scintillator liquid NE-213 of the DEMON cells. In particular the cross talk between detectors, when a neutron is scattered from one detector to another thereby simulating a two-neutron event, had to be investigated. The effect is largest for neighbouring detectors, thus the neutron-neutron angular distributions are mainly affected for small relative neutron angles. Primarily the simulations served for testing the accuracy and credibility of the analysis procedure as described in the next chapter. Of major concern was the structures introduced in the angular distributions by the modular setup of 60 neutron detectors.

The simulations are based on the GEANT4 toolkit [11] and the MENATE-R physics list [12,13]. Physical input data for fragment velocities and neutron multiplicities were taken from Ref. [14]. The distribution of multiplicities was assumed to be a 2D-normal distribution. Neutron energies $\eta$ in the cms were selected randomly from a Maxwellian distribution

$$
\varphi(\eta) \sim \sqrt{\eta} e^{-\eta / T}
$$

with $T$ the temperature of the daughter nucleus. The kinematical focusing was calculated by adding the fragment velocity to the neutron velocity from Eq. (2). For each two-neutron fission event the relative angle between the neutrons is computed. Taking into account kinematical focusing, the isotropic distribution in the $\mathrm{cms}$ of fragments becomes forward-backward asymmetric in the laboratory.

The dynamical anisotropy was introduced by assuming the fission fragments to have large angular momenta $J$ up to $J \approx 8 \hbar$. Angular momenta are aligned perpendicular 

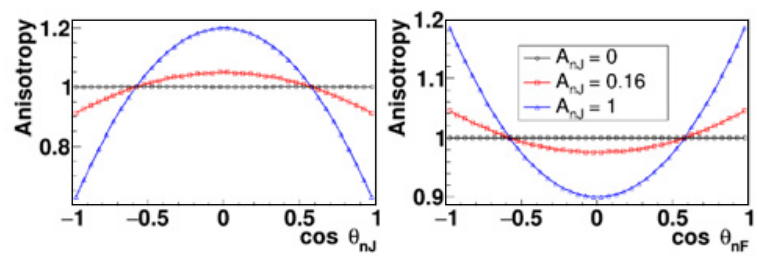

Figure 4. Simulated neutron angular distribution of $\theta_{n F}$ relative to the fission axis plotted as function of $\cos \theta_{n F}$ for various anisotropies $A_{n J}$ in Eq. (4). The normalized distribution reads $W\left(\theta_{n F}\right) \sim 1+b\left(3 \cos ^{2} \theta_{n F}-1\right) / 2$ with $b=2 A_{n F} /(3+$ $\left.A_{n F}\right)$. From theory the anisotropy $A_{n F} \approx 0.08$ is evaluated which for the normalized distribution yields $\mathrm{b} \approx 0.052[10,16]$.

to the fission axis [15]. Neutrons evaporated from a rotating nucleus will preferentially be emitted in a plane perpendicular to nuclear angular momentum (see Fig. 2). This anisotropy is parameterized by

$$
W\left(\theta_{n J}\right)=1+A_{n J} \sin ^{2} \theta_{n J}
$$

where $\theta_{n J}$ is the neutron angle relative to fragment spin and $A_{n J}$ the anisotropy parameter $A_{n J}=\frac{W\left(90^{0}\right)-W\left(0^{0}\right)}{W\left(0^{0}\right)}$. Since fragment spins are not fixed in space but aligned in a plane perpendicular to the fission axis, the anisotropy of neutron emission relative to the fission axis is obtained by averaging over spin $J$ in the plane perpendicular to the fission axis as

$$
W\left(\theta_{n F}\right)=1+A_{n F} \cos ^{2} \theta_{n F}
$$

where $\theta_{n F}$ is the neutron angle relative to the fission axis and $A_{n F}$ the anisotropy parameter $A_{n F}=\frac{W\left(0^{0}\right)-W\left(90^{\circ}\right)}{W\left(90^{\circ}\right)}$. For perfect spin alignment the relation between the two anisotropy parameters $A_{n J}$ and $A_{n F}$ is $A_{n F} \approx A_{n J} / 2$ $[6,10]$.

The simulated angular neutron distribution $W\left(\theta_{n F}\right)$ from Eq. (4) is plotted in Fig. 4 for different anisotropy parameters $A_{n J}$. Theory suggests $A_{n F} \approx 0.08 \quad[10,16]$, which corresponds to $A_{n J} \approx 0.16$. Practically the same magnitude of anisotropy was concluded from experiment [3]. Taking $A_{n J}=1$ clearly overestimates the effects and only serves the purpose of demonstration. Note that these distributions are calculated in the cms of fragments and cannot be observed in experiment. The distributions which are accessible to experiment are the neutronfragment and the neutron-neutron angular correlations. In the present simulations they were also evaluated and are shown in Fig. 5. From the simulations it is quite obvious that in the laboratory system the dynamical anisotropy is overwhelmed by the kinematical anisotropy and barely visible. By contrast, in the new type of experiments proposed in the present work, measuring neutron-neutron-fragment events and projecting them on a plane perpendicular to the fission axis, the dynamical anisotropy can be identified in the projection by a structure in the distribution of relative angles $\Phi_{n n}$ parameterized in Eq. (1) and sketched in the bottom part of Fig. 3. The challenge here is, however, the small size of the anisotropy anticipated.

\section{Analysis and results}

The main problem in the analysis of data from experiment with many detectors, each of them having its own

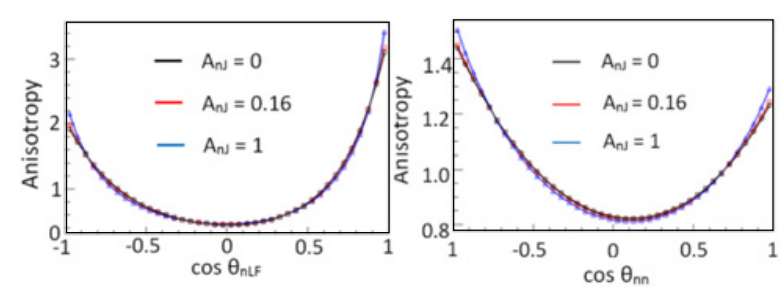

Figure 5. Simulated angular distributions in the laboratory system of the angle $\theta_{n L F}$ between neutron and Light Fragment (left figure) for different anisotropy parameters $A_{n J}$ from Eq. (3). Simulated angular distributions in the laboratory system of the angle $\theta_{n n}$ between two neutrons (right figure) for different anisotropy parameters $A_{n J}$ in Eq. (3). The size of the anisotropy $A_{n J}=0.16$ is close to theoretical prediction [10].

geometrical and intrinsic efficiency, is how to take into account different detector efficiencies. We used a procedure of self-normalization of the neutron-neutron angular distributions. The procedure was tested in the simulations and proved to be accurate. It consists in building "pseudo-random" neutron-neutron angular distributions with two neutrons originating from different fission events. This can be either the distribution of the relative angle between two neutrons (as shown in Fig. 5, right) or the projection of the angular distribution onto the $X Y$ plane (see Fig. 3, bottom). In the pseudorandom approach the geometrical and intrinsic efficiencies of the neutron detectors are considered, but the actual correlations between two neutrons are missing. So making such a "pseudo-random" angular distribution is practically equivalent to the simulation of the neutron-neutron angular distributions with zero correlation, but with very accurate detector response, taken from the experimental data. By normalizing the measured neutron-neutron angular distribution to such a "pseudo-random" one, we obtain the distribution, which is free of the geometrical effects. There are several problems, however, which still remain not fully solved after such normalization. One of them is the crosstalk between different detectors. If the cross-talk is large enough, it may distort the resulting distribution. Another problem arises from the finite angular resolution of the fission fragment detector CODIS. It influences in a slightly different way the true neutron-neutron and the "pseudorandom" distributions where the two neutrons come from different fission events. This may produce an additional small structure, distorting the effect sought.

In order to minimize the influence of these factors, several conditions were imposed in the data analysis: 1) only events with light fragments emitted within a narrow cone around a chamber axis were selected $\left(\cos \theta_{L F}>0.95\right.$ with $\theta_{L F}$ being the cone's half-angle, see Fig. 6) to ensure accurate determination of the fission axis direction; 2) the relative angle between two neutrons was required to be $\theta_{n n}>65^{\circ}$, and 3) the time-of-flight TOF of neutrons was limited to TOF $<10 \mathrm{~ns}$. The two latter restrictions virtually eliminated the cross-talk between neighboring DEMON detectors.

Figure 6 shows different steps of analysis using the "pseudo-random" normalization method. The measured (a) and "pseudo-random" (b) distributions are close to each other as to be expected due to the small size anticipated for the dynamical anisotropy. The resulting normalized distribution for the case of $\cos \theta_{L F}>0.95$ is shown in 

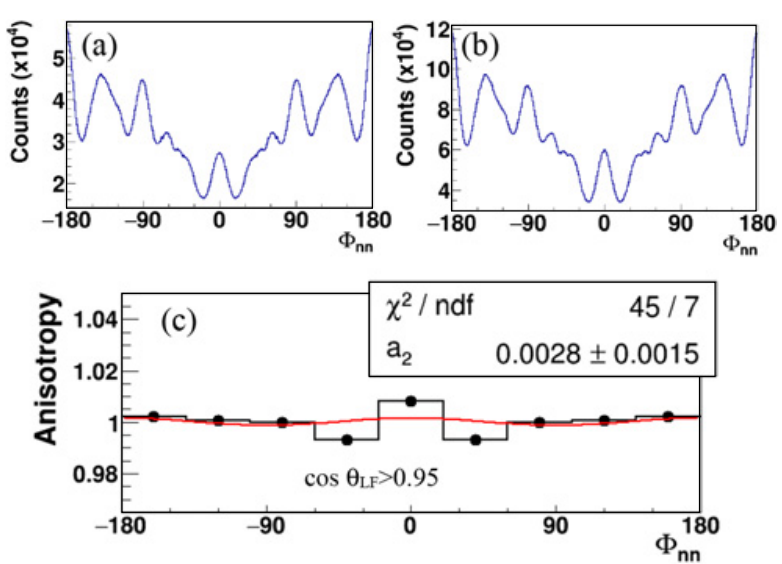

Figure 6. (a) Experimental neutron-neutron angular distribution, projected onto a plane, perpendicular to the fission axis; (b) "pseudo-random" distribution with neutrons from different events; (c): the ratio of the experimental and "pseudo-random" distributions for the angles of the fission axis relative to the CODIS chamber axis $\cos \theta_{L F}>0.95$.

Fig. 6(c) together with the result of a least-square fit by the formula (1). Applying the statistical errors as weights, results in a non-zero anisotropy parameter of $\boldsymbol{a}_{2}=(2.8 \pm 1.5) \cdot 10^{-3}$. However, the $\chi^{2} / n$ value of the fit is rather high, which indicates that systematic errors are still dominating the statistical ones. The total error is estimated to be at the order of $\mathbf{4} \times \mathbf{1 0}^{-\mathbf{3}}$. In view of this large uncertainty, one has to conclude that at the present stage of analysis the dynamical asymmetry is neither excluded nor definitely proven to exist within the limits of experimental accuracy.

\section{Conclusion}

The theoretically predicted effect of the neutron-neutron angular correlations in the plane perpendicular to the fission axis has been searched for in a multi-parameter experiment using a set of 60 DEMON neutron detectors and the angle-sensitive fission fragment detector CODIS. The effect hasn't been clearly found so far due to large systematic uncertainties, although the statistical accuracy would be sufficient for its observation. Currently, further improvements in the data analysis and related simulations are in process, e.g. by taking advantage of the large angular acceptance of the CODIS fission chamber, in order to circumvent the present problem of systematic accuracy.
For the time being the CORA method is the only one which is exclusively sensitive to the dynamical anisotropy of neutron emission in the cms of fragments. The $(n, n, F)$ data taken allow simultaneously analysing $(n, F)$ correlations in the search of scission neutrons [16] provided the dynamical anisotropy is known otherwise, e.g. from the present type of experiments.

This work was partially supported by the JINR-IN2P3 agreement, which is gratefully acknowledged.

\section{References}

[1] H.R. Bowman, S. Thompson, J.C.D. Milton, and W.J. Swiatecki, Phys. Rev. 126, 2120 (1962)

[2] K. Skarsvåg, and K. Bergheim, Nucl. Phys. 45, 72 (1963)

[3] A.S. Vorobyev, O.A. Shcherbakov, A.M. Gagarski, G.V. Val'ski, and G.A. Petrov, EPJ Web of Conferences 8, 03004 (2010)

[4] N. Carjan, and M. Rizea, Phys. Lett. B 747, 178 (2015)

[5] A. Gavron, Phys. Rev. C 13, 2562 (1976)

[6] V.E. Bunakov, I.S. Guseva, S.G. Kadmensky, and G.A. Petrov, Bull. Rus. Acad. Sci. Phys. 70, 1853 (2006)

[7] Yu.N. Kopach, P. Singer, M. Mutterer, M. Klemens, A. Hotzel, D. Schwalm, P. Thirolf, M. Hesse, and F. Gönnenwein, Phys. Rev. Lett. 82, 303 (1999)

[8] Yu.N. Kopatch, M. Mutterer, D. Schwalm, P. Thirolf, and F. Gönnenwein, Phys. Rev. C 65, 044614 (2002)

[9] I. Tilquin, El Masri, M. Parlog, et al., NIM A 365, 466 (1995)

[10] I. Guseva, ,Proc. Int. Seminar ISINN-23, May 25-29, 2015, Dubna, Russia, JINR, E3-2016-12, p. 80 (2016)

[11] S. Agostinelli, J. Allison, K. Amako, et al., NIM A 506, 250 (2003)

[12] P. Desesquelles, A.J. Cole, A. Dauchy, et al., NIM A 307, 366 (1991)

[13] B. Roeder, EURISOL Design Study, 38 (2008)

[14] H. Henschel, A. Kohnle, H. Hipp, and F. Gönnenwein, NIM 190, 125 (1981)

[15] J.B. Wilhelmy, E. Cheifetz, R.C. Jared, S.G. Thompson, H.R. Bowman, and J.O. Rasmussen, Phys. Rev. C 5, 2041 (1972)

[16] A. Chietera, PhD Thesis "Angular correlations between fragments and neutrons in the spontaneous fission of 252Cf", Strasbourg University, 2015 